\title{
Características sociodemográficas y conocimientos sobre la prevención de cáncer gástrico en usuarios de un centro de salud del Perú
}

\author{
Sociodemographic characteristics and knowledge of gastric cancer
} prevention in users of a health center in Peru

\author{
Flores-Blancas, Jakeline ${ }^{1}$ \\ Ortiz-Romaní, Katherine Jenny ${ }^{2}$
}

\begin{abstract}
Resumen
Objetivo: determinar la asociación entre las características sociodemográficas y el conocimiento sobre la prevención de cáncer gástrico en usuarios de un centro de salud. Materiales y métodos: estudio fue descriptivo-correlacional de corte transversal. La muestra fue de 118 usuarios que se atendían en un centro de salud de Lima-Perú, 2018. Las variables del estudio fueron los conocimientos sobre la prevención del cáncer gástrico y las características sociodemográficas. Se aplicaron las pruebas de t de Student y ANOVA para el análisis inferencial. Resultados: la media del conocimiento sobre la prevención del cáncer gástrico fue 8.7. Las características sociodemográficas asociadas al conocimiento fueron el nivel educativo $(\mathrm{p}=0.05)$ y el tipo de seguro de salud $(\mathrm{p}=0.05)$. Las medias con menor puntuación se observan en primaria (media=7.9) seguro integral de salud (media=8.5). Respecto a la asociación entre las características con la dimensión factor biológico, sólo se encontró con la Región ( $\mathrm{p}=0.01$ ) y la media de menor puntaje de esta dimensión la obtuvieron usuarios naturales de la Sierra. Conclusión: los conocimientos sobre la prevención del cáncer gástrico se asocian con el nivel educativo, el tipo de seguro de salud, y respecto a la dimensión factor biológico se asocia con la región natural de procedencia. Menores puntajes los obtienen quienes tienen un nivel educativo primaria, un seguro integral de salud y los de origen Sierra. Por ello, el personal de enfermería debe realizar intervenciones de promoción y prevención de esta patología a la comunidad.
\end{abstract}

Palabras clave: Prevención de enfermedades; Neoplasias gástricas; Características de la población (Fuente: DeCS).

\begin{abstract}
Objective: to determine the association between sociodemographic characteristics and knowledge about gastric cancer prevention in users of a health center. Materials and methods: study was descriptive-correlational in cross section. The sample was 118 users who were treated at a health center in Lima-Peru, 2018. The variables of the study were knowledge of gastric cancer prevention and sociodemographic characteristics. Student's t-tests and ANOVA were applied for inferential analysis. Results: the mean knowledge about gastric cancer prevention was 8.7. The sociodemographic characteristics associated with knowledge were educational level $(\mathrm{p}=0.05)$ and type of health insurance $(\mathrm{p}=0.05)$. The lowest scoring means are observed in primary (mean=7.9) comprehensive health insurance (mean=8.5). Regarding the association between the characteristics with the biological factor dimension, it was only found with the Region ( $\mathrm{p}=0.01$ ) and the mean with the lowest score of this dimension was obtained from natural users of the Sierra. Conclusion: knowledge about gastric cancer prevention is associated with educational level, type of health insurance, and regarding the biological factor dimension, it is associated with the natural region. Lower scores are obtained by those who have a primary educational level, comprehensive health insurance and those of Sierra origin. For this reason, nursing personnel must carry out interventions to promote and prevent this pathology in the community.
\end{abstract}

Keys words: Disease prevention; Gastric neoplasms; Population characteristics (Source: DeCS).

Para citar:

Flores J, Ortiz KJ. Características sociodemográficas y conocimientos sobre la prevención de cáncer gástrico en usuarios de un centro de salud del Perú. CASUS. 2020;5(1);1-7.

DOI: $10.35626 /$ casus. 1.2020 .240

${ }^{1}$ Bachiller en Enfermería. Hospital Cayetano Heredia.

${ }^{2}$ Licenciada en Enfermería. Departamento de investigación de la Facultad de Ciencias de la Salud de la Universidad Católica Sedes Sapientiae.

Correo electrónico: jakeline.flores96@gmail.com
Fecha de recepción: 15-01-20

Fecha de envío a pares: 16-01-20

Fecha de aprobación por pares: 12-03-20

Fecha de aceptación: 13-03-20 


\section{INTRODUCCIÓN}

El cáncer gástrico (CG) es un problema que afecta a la salud pública y es la tercera causa de decesos por cáncer en ambos sexos a nivel mundial con 782685 muertes al año (1). A nivel nacional, en el año 2018, el CG ocupó el tercer lugar de todos los cánceres y se registraron 4606 muertes (1). Durante el periodo 2010-2012 se estimó que fue la primera causa de muerte en Lima Metropolitana reportándose 3437 muertos y con tasa de mortalidad de 12 por cada 100000 habitantes (2). Asimismo, la infección del Helicobacter pylori es el principal factor de riesgo y la principal causa de mortalidad; seguido de los factores dietéticos y ambientales (3).

En una etapa temprana, el CG puede ser clínicamente silencioso y, por ello, la mayoría de los pacientes se percata que tiene cáncer en etapas avanzadas. Por lo contrario, si se diagnostica tempranamente las tasas de supervivencia a 5 años superan el $90 \%$ (4). No obstante, diagnosticado en una etapa avanzada, las tasas de supervivencia a 5 años pueden estar en el rango de 10 a 20\% (5). Actualmente, la endoscopia gastrointestinal es una herramienta indispensable en el campo gastroenterológico, ya que ha avanzado su diagnóstico y tratamiento. Sin embargo, hay personas que desconocen de este examen o no tienen los recursos económicos para poder realizarlo (6).

Existen pocos estudios acerca del conocimiento sobre la prevención del cáncer gástrico y este fenómeno ha sido vinculado a la falta de promoción y prevención de esta patología (7-13). En el Perú existe una serie de dificultades como un diagnóstico tardío, en el acceso a servicios de salud, los altos costos de un despistaje como la endoscopia, la falta de cultura de prevención, los estilos de vida saludables, la falta de un seguro de salud, el tener un bajo nivel educativo, la pobreza, entre otros asociados una alta tasa de mortalidad por CG (14).

Considerando estas altas tasas de morbimortalidad, en países en desarrollo como el Perú, a fin de poder reducirlas se debería tomar en cuenta a las personas vulnerables a este cáncer. Con el fin de que ellos conozcan tempranamente los factores de riesgo ambientales modificables, como la dieta y la infección por Helicobacter pylori (12). Asimismo, la participación de los profesionales de la salud podría influenciar en la reducción de esta problemática. Si bien es cierto existen estudios sobre los factores de riesgo asociados al cáncer gástrico $(15,16)$, aún hay escasez de estudios sobre la prevención y promoción de esta enfermedad y el perfil sociodemográfico de los usuarios que aún no tienen esta patología. Es por ello, que el objetivo del estudio fue determinar la asociación entre las características sociodemográficas y el conocimiento sobre la prevención de cáncer gástrico en usuarios de un centro de Salud.

\section{MATERIALES Y MÉTODOS}

El estudio fue descriptivo-correlacional de corte transversal. Para poder calcular la muestra los criterios fueron: una proporción próxima al 10\%, con un intervalo de confianza del $95 \%$ y un margen de error de 0.05 en poblaciones infinitas. El tamaño de la muestra calculado fue de 139 usuarios de ambos sexos igual o mayores de 18 años que acudieron a un centro de salud público de primer nivel de atención de la ciudad Lima-Norte, 2018. El muestreo fue no probabilístico por conveniencia. Sin embargo, se logró recolectar solo una muestra de $n=118$, porque los usuarios no llenaron correctamente los cuestionarios y otros no desearon participar en el estudio.

Para medir el conocimiento sobre la prevención de cáncer gástrico (CPCG) se empleó un cuestionario. El mismo fue sometido a jueces expertos y la prueba binomial con resultados válidos $(<0.05)$. Asimismo, para la confiabilidad fue sometido a una prueba piloto y el resultado del coeficiente de alfa de Cronbach arrojó un valor de 0.84 (17). El cuestionario constó de 15 preguntas y para la evaluación de cada una de ellas sólo se tomó en cuenta, de las cuatro alternativas, una respuesta correcta. Cabe resaltar que la variable fue de naturaleza cuantitativa, con un rango total de 0-15 puntos y constó de tres dimensiones. La primera dimensión, titulada factor genético personal, constó de 5 preguntas con un rango de 0 a 5 puntos 
referentes a sexo y edad predisponente a tener cáncer gástrico, antecedentes de cáncer en familiares, historial clínico de enfermedades precursoras y tipo de trabajo. La segunda dimensión fue el factor medioambiental con siete preguntas, con un rango de 0 a 7 puntos, constó sobre alimentación, higiene y hábitos nocivos como el tabaco. La tercera dimensión fue el factor biológico de tres preguntas, con un rango de 0 a 3 puntos, sobre la infección por Helicobacter pylori.

Para la evaluación de las características sociodemográficas se empleó una ficha de datos de cinco preguntas acerca de la edad (años); el sexo (masculino y femenino); nivel educativo (primaria, secundaria y superior); la ocupación actual (sin trabajo y con trabajo) y el tipo de seguro (seguro integral de salud (SIS) y Essalud (seguro social de salud) y privado (otros seguros privados). Cabe resaltar que el SIS es un organismo público ejecutor del Ministerio de Salud del Perú para las poblaciones vulnerables y de situaciones de pobreza (18). Asimismo, Essalud es un seguro social de salud y un organismo público descentralizado afiliado al Ministerio de Trabajo para los que tienen un trabajo formal (19).

Para el análisis estadístico se utilizó el programa Stata versión 14. Se realizó una estadística descriptiva mediante frecuencias y porcentajes, para las variables categóricas. De igual manera, para las variables numéricas se utilizaron la media y la desviación estándar. Respecto al análisis inferencial para la asociación de una variable numérica vs una categórica se determinó en primera instancia la normalidad de la variable numérica mediante la prueba de KolmogórovSmirnov, para emplear las pruebas de $t$ de Student y ANOVA y la prueba de Spearman con una significancia estadística menor o igual a 0.05 .

Para la recolección de datos se contó con la aprobación del Comité de Ética de Investigación en Salud de la Universidad Católica Sedes Sapientiae y el consentimiento por parte de los encuestados. Asimismo, los resultados obtenidos se mantuvieron en anonimato. Es importante señalar que en el presente estudio no hubo riesgos para la salud debido a las características del estudio.

\section{RESULTADOS}

De los 118 encuestados la edad media en años fue 36.6. Asimismo, del total, la mayoría fueron mujeres $(75.4 \%)$, tenían educación secundaria (56\%), contaban con SIS (89\%) y no tenían un trabajo (72\%). La media del conocimiento sobre la prevención del cáncer gástrico fue 8.7 (ver tabla $1)$.

Tabla 1. Descripción de la muestra

\begin{tabular}{|c|c|c|}
\hline & $\mathbf{n}$ & $(\%)$ \\
\hline Edad (Media \pm DE) & \multicolumn{2}{|c|}{$38.6 \pm 6.8$} \\
\hline \multicolumn{3}{|l|}{ Sexo } \\
\hline Masculino & 29 & $24.6 \%$ \\
\hline Femenino & 89 & $75.4 \%$ \\
\hline \multicolumn{3}{|l|}{ Nivel educativo } \\
\hline Primaria & 26 & $22.0 \%$ \\
\hline Secundaria & 66 & $56.0 \%$ \\
\hline Superior & 26 & $22.0 \%$ \\
\hline \multicolumn{3}{|l|}{ Sistema de salud } \\
\hline Seguro integral de salud & 105 & $89.0 \%$ \\
\hline Essalud y privado & 13 & $11.0 \%$ \\
\hline \multicolumn{3}{|l|}{ Ocupación actual } \\
\hline Sin trabajo & 85 & $72.0 \%$ \\
\hline Con trabajo & 33 & $28.0 \%$ \\
\hline \multicolumn{3}{|l|}{ Religión } \\
\hline Católico & 75 & $63.6 \%$ \\
\hline Evangélico & 22 & $18.6 \%$ \\
\hline Otros & 21 & $17.8 \%$ \\
\hline Conocimiento (Media \pm DE) & \multicolumn{2}{|c|}{$8.7 \pm 2.2$} \\
\hline Factor genético personal* & \multicolumn{2}{|c|}{$1.9 \pm 0.9$} \\
\hline Factor medioambiental* & \multicolumn{2}{|c|}{$5.8 \pm 1.2$} \\
\hline Factor biológico* & \multicolumn{2}{|c|}{$0.9 \pm 1.3$} \\
\hline
\end{tabular}

En la tabla 2 se evidenció que el conocimiento sobre la prevención del cáncer gástrico se asoció significativamente con el nivel educativo $(\mathrm{p}=0.05)$ $y$ el tipo de seguro de salud $(\mathrm{p}=0.05)$. El promedio más alto de CPCG corresponde al nivel educativo superior (media $\pm \mathrm{DE}=9.3 \pm 1.9$ ) en comparación con los que tienen primaria (media $\pm \mathrm{DE}=7.9 \pm 2.5$ ). 
Asimismo, los valores más altos del CPCG corresponden a tener un seguro de Essalud y privado (media $\pm \mathrm{DE}=9.8 \pm 2.0$ ) en comparación con los que tienen SIS (media $\pm \mathrm{DE}=8.5 \pm 2.2$ ). El resto de las asociaciones no fueron significativas.

Respecto a la asociación entre los factores sociodemográficos y las dimensiones del CPG, solo se encontró asociación entre región y la

Tabla 2. Asociación descriptiva entre los factores sociodemográficos y conocimientos sobre la prevención de cáncer gástrico

\begin{tabular}{|c|c|c|}
\hline \multirow{2}{*}{$\begin{array}{l}\text { Características } \\
\text { sociodemográficas }\end{array}$} & \multicolumn{2}{|c|}{$\begin{array}{c}\text { Conocimiento sobre la } \\
\text { prevención de cáncer } \\
\text { gástrico }\end{array}$} \\
\hline & $($ Media \pm DE $)$ & $\mathbf{p}$ \\
\hline Edad* & -0.03 & 0.74 \\
\hline \multicolumn{3}{|l|}{ Sexo } \\
\hline Hombre & $8.6 \pm 2.2$ & 0.87 \\
\hline Mujer & $8.7 \pm 2.2$ & \\
\hline \multicolumn{3}{|l|}{ Nivel educativo } \\
\hline Primaria & $7.9 \pm 2.5$ & 0.05 \\
\hline Secundaria & $8.7 \pm 2.0$ & \\
\hline Superior & $9.3 \pm 1.9$ & \\
\hline \multicolumn{3}{|l|}{ Sistema de salud } \\
\hline Seguro integral de salud & $8.5 \pm 2.2$ & 0.05 \\
\hline Essalud y privado & $9.8 \pm 2.0$ & \\
\hline \multicolumn{3}{|l|}{ Ocupación actual } \\
\hline Sin trabajo & $8.8 \pm 2.1$ & 0.43 \\
\hline Con trabajo & $8.4 \pm 2.3$ & \\
\hline Religión & & 0.56 \\
\hline Católico & $8.8 \pm 2.1$ & \\
\hline Evangélico & $8.4 \pm 2.4$ & \\
\hline Otros & $8.4 \pm 2.1$ & \\
\hline Región natural & & 0.18 \\
\hline Costa & $9.0 \pm 2.0$ & \\
\hline Sierra & $8.3 \pm 2.3$ & \\
\hline Selva & $8.9 \pm 2.2$ & \\
\hline
\end{tabular}

*Correlación con la prueba Spearman.

dimensión factor biológico ( $\mathrm{p}=0.01)$, es decir que los usuarios naturales de la región Sierra tuvieron un promedio inferior (media $\pm \mathrm{DE}=0.6 \pm 1.1$ ) de conocimiento sobre el Helicobacter pylori en contraste con los que fueron naturales de la Costa (media $\pm \mathrm{DE}=1.3 \pm 1.4)$ (ver tabla 3$)$.

\section{DISCUSIÓN}

En el presente estudio se observa que el promedio del conocimiento sobre la prevención del cáncer gástrico fue 8.7. Este resultado considera que los encuestados tenían un conocimiento medio. Entre los hallazgos se muestra la asociación entre el nivel educativo, el sistema de salud con el CPCG. Los usuarios que tuvieron valores inferiores de conocimiento fueron los que tenían educación inferior SIS y pertenecieron a la región Sierra.

Un hallazgo importante del estudio fue que el promedio inferior de CPCG correspondió al nivel educativo secundaria y primaria de los usuarios en comparación con los que tuvieron nivel superior. Estudios nacionales reportaron que las personas con niveles superiores de educación tenían un conocimiento alto sobre cáncer en general $(8,9)$.

Por otro lado, un estudio realizado en Colombia demostró que usuarios de consultorios externos de un establecimiento de salud con secundaria incompleta no conocían cómo prevenir el CG en comparación con los que tuvieron un nivel educativo superior (10). El tener un nivel de educación superior crea en la persona nuevos conocimientos y maneras de proporcionar explicaciones, formular pronósticos, entre otros.

Además, en niveles educativos altos es frecuente que se empleen las tecnologías de información y la comunicación (TIC) que perfeccionan la adquisición de un buen nivel de conocimiento sobre un tema específico. En este caso de un cáncer u otras patologías (11). Lamentablemente, los usuarios que tienen niveles educativos inferiores no tienen esa oportunidad debido a la pobreza y escasez de información y por consiguiente son más vulnerables a la enfermedad y a un incremento de riesgo de mortalidad $(20,21)$.

Asimismo, el conocimiento sobre la prevención del CG se asoció significativamente con el tipo de seguro de salud. Los valores más bajos del CPCG corresponden a los que cuentan con SIS en comparación con los que tienen Essalud y seguro privado. Un estudio en Perú reportó que en un grupo de usuarias que tenían un seguro gratuito o subsidiado tenían un conocimiento deficiente sobre el cáncer (8). Esto podría explicarse porque los usuarios que poseen SIS son una población vulnerable y la mayoría de ellos no tiene acceso a una educación y por ende desconocen de muchas enfermedades (12). Actualmente, en el Perú más 
del 50\% de pacientes con algún tipo de cáncer son atendidos de manera gratuita por medio del SIS para tratar su enfermedad (13) no obstante, esta ayuda es necesaria pero no disminuirá la tasa de morbimortalidad del cáncer. Lo que se necesita para disminuir esta tasa es una cultura de promoción y prevención de la salud por parte del personal de salud mediante sesiones oportunas para diagnosticar precozmente los cánceres y cambiar conductas inadecuadas de la población por falta de educación (22).

Otro resultado del estudio fue que los usuarios naturales de la región Sierra tienen promedios bajos de la dimensión factor biológico de conocimientos sobre el cáncer gástrico

Tabla 3. Asociación entre los factores sociodemográficos y las dimensiones y conocimientos sobre la prevención del cáncer gástrico

\begin{tabular}{|c|c|c|c|c|c|c|}
\hline \multirow[b]{2}{*}{ Características sociodemográficas } & \multicolumn{2}{|c|}{$\begin{array}{c}\text { Factor genético } \\
\text { personal }\end{array}$} & \multicolumn{2}{|c|}{$\begin{array}{c}\text { Factor } \\
\text { medioambiental }\end{array}$} & \multicolumn{2}{|c|}{ Factor biológico } \\
\hline & (Media \pm DE) & $\mathrm{p}$ & $($ Media \pm DE) & $\mathrm{p}$ & $($ Media \pm DE) & $\mathrm{p}$ \\
\hline Edad* & -0.02 & 0.80 & 0.1 & 0.30 & -0.1 & 0.30 \\
\hline \multicolumn{7}{|l|}{ Sexo** } \\
\hline Hombre & $1.9 \pm 1.1$ & 0.90 & $5.7 \pm 1.4$ & 0.60 & $0.9 \pm 1.4$ & 0.80 \\
\hline Mujer & $1.9 \pm 0.1$ & & $5.9 \pm 1.1$ & & $0.8 \pm 1.3$ & \\
\hline \multicolumn{7}{|l|}{ Nivel educativo $* * *$} \\
\hline Primaria & $1.8 \pm 1.0$ & 0.80 & $5.6 \pm 1.5$ & 0.90 & $0.5 \pm 1.1$ & 0.08 \\
\hline Secundaria & $1.9 \pm 0.9$ & & $5.8 \pm 1.1$ & & $0.9 \pm 1.4$ & \\
\hline Superior & $2.1 \pm 0.9$ & & $5.9 \pm 1.0$ & & $1.3 \pm 1.4$ & \\
\hline \multicolumn{7}{|l|}{ Sistema de salud } \\
\hline Seguro integral de salud & $1.93 \pm 0.9$ & 0.60 & $5.8 \pm 1.2$ & 0.20 & $0.8 \pm 1.3$ & 0.10 \\
\hline Essalud y privado & $2.07 \pm 1.0$ & & $6.2 \pm 1.0$ & & $1.4 \pm 1.5$ & \\
\hline \multicolumn{7}{|l|}{ Ocupación actual } \\
\hline Sin trabajo & $2.0 \pm 0.9$ & 0.20 & $5.9 \pm 1.2$ & 0.60 & $0.8 \pm 1.3$ & 0.90 \\
\hline Con trabajo & $1.7 \pm 1.1$ & & $5.7 \pm 1.3$ & & $0.9 \pm 1.4$ & \\
\hline \multicolumn{7}{|l|}{ Religión } \\
\hline Católico & $2.1 \pm 0.9$ & 0.30 & $5.8 \pm 1.2$ & 0.90 & $0.9 \pm 1.4$ & 0.70 \\
\hline Evangélico & $1.8 \pm 0.8$ & & $5.8 \pm 1.4$ & & $0.8 \pm 1.4$ & \\
\hline otros & $1.7 \pm 1.1$ & & $5.9 \pm 1.1$ & & $0.7 \pm 1.2$ & \\
\hline \multicolumn{7}{|l|}{ Región natural } \\
\hline Costa & $1.9 \pm 0.9$ & 0.30 & $5.8 \pm 1.1$ & 0.90 & $1.3 \pm 1.4$ & 0.01 \\
\hline Sierra & $1.8 \pm 0.9$ & & $5.9 \pm 1.4$ & & $0.6 \pm 1.1$ & \\
\hline Selva & $2.3 \pm 1.1$ & & $5.9 \pm 1.4$ & & $0.8 \pm 1.3$ & \\
\hline
\end{tabular}

comparados a los de la región Costa. Aunque no se reportan investigaciones con resultados similares, se conoce que los peruanos que viven en la región Sierra son más propensos a presentar cáncer (23) porque dentro de las regiones más pobres del Perú, geográficamente, se considera a la Sierra y, por ende, el factor económico está relacionado con un mayor acceso a la salud (23).

Entre las limitaciones de estudio se debe considerar que, por la falta de participación de los usuarios, la población fue reducida. Asimismo, se pudo considerar otras características de la población como, por ejemplo: laborales, profesionales, antecedentes patológicos personales o familiares, etc. Entre las limitaciones del diseño figura el sesgo de memoria y de selección. Es por ello, que se recomienda incluir en futuros estudios muestras más amplias considerando un mayor número de factores.

\section{CONCLUSIONES}

El promedio del conocimiento sobre la prevención del CG es 8.7 y se muestra que hay asociación entre el CPCG y el nivel educativo. Los valores más bajos del conocimiento corresponden a los que tienen nivel educativo primaria y secundaria. Asimismo, el tipo de seguro de salud con valores más bajos de conocimiento que corresponden a los que tienen SIS. Además, los que residieron de la 
región Sierra tienen promedios bajos de la dimensión factor biológico de CPCG, en comparación a los de la región Costa. Estos hallazgos indican la urgencia de la creación y ejecución de programas educativos acerca del CG, por parte del personal de enfermería y de salud en general de las postas y los centros de salud en poblaciones con este perfil sociodemográfico. A fin de aumentar los conocimientos de sobre la enfermedad y mejorar la comunicación enfermerousuario. Finalmente, es importante que se tenga en cuenta el contenido de las charlas para una fácil comprensión de los usuarios, así como los términos empleados en las pruebas de evaluación de conocimientos sobre este tipo de cáncer.

\section{AGRADECIMIENTOS}

A la Lic. Carolina Chinchay Quispe por el apoyo en la recolección de datos.

\section{REFERENCIAS BIBLIOGRÁFICAS}

1. Global Cancer Observatory. Stomach Source 2018 [Internet]. París, Francia: GLOBOCAN; 2018 [citado el 01 de diciembre de 2019] Disponible en: https://gco.iarc.fr/today/data/factsh eets/cancers/7-Stomach-factsheet.pdf

2. Instituto Nacional de Enfermedades Neoplásicas. Registro de cáncer de Lima metropolitana incidencia y mortalidad 2010 - 2012 [Internet] Lima, Perú: INEN; 2016 [citado el 07 de diciembre de 2019] Disponible en: http://www.inen.sld.pe/portal/docu mentos/pdf/banners_2014/2016/Re gistro\%20de\%20C\%C3\%A1ncer $\% 20$ Lima\%20Metropolitana\%202 010\%20-\%202012_02092016.pdf

3. Liu Q, Zeng X, Wang W, Huang $\mathrm{R}$, Huang $\mathrm{Y}$, Liu $\mathrm{S}$, et al. Awareness of risk factors and warning symptoms and attitude towards gastric cancer screening among the general public in China: a cross-sectional study. BMJ Journals. 2019; 9(7):1-9.

4. Piazuelo M, Correa P, Cáncer Gástrico: Punto de vista. Colombia Médica. 2013;44(3)192-201.

5. Ries LAG, Kosary CL, Hankey 9 BF, Miller BA, Harras A, Edwards
BK. Cancer Statistics Review 1973-1994, National Cancer Institute, NIH Publication No. 972789. Bethesda: Department of Health and Human Services, 1997.

. Tajiri H, Niwa H. Proposal for a consensus terminology in endoscopy: how should different endoscopic imaging techniques be grouped and defined? Endoscopy. 2008;40(9):775-778.

7. Bazán C. Conocimiento y prácticas relacionados con la prevención y diagnóstico del cáncer de cuello uterino, en usuarias aseguradas al S.I.S. que acuden a consultorio externo del hospital Santa María de Cutervo [Tesis de Maestría en salud pública]. Lambayeque: Universidad nacional Pedro Ruiz Gallo; 2017.

Dávila G, Factores socioeconómicos y su relación con el nivel de conocimiento sobre prevención del cáncer en el servicio de hospitalización de gineco obstetricia del hospital de Satipo en el tercer trimestre de 2015 [Tesis de Maestría en salud pública]. Huánuco: Universidad Nacional Hermilio Valdizán De Huánuco; 2016.

Muñoz E, Díaz J, Shoemaker R, Ordoñez R, Nino L, Bustamante
A, et al. Grado de conocimiento acerca del cáncer gástrico en popayán, Colombia. Revista Facultad Ciencias de la Salud. 2012;14(3):9-14

10. Hughes P. La contribución de la educación superior al sistema educativo en su conjunto. Conferencia Mundial sobre la Educación Superior [Internet]. París, Francia: [citado el 25 enero del 2019]. Disponible en: http://www.unesco.org/education/e ducprog/wche/principal/systems.html

11. Vergara G. Aproximar el sector salud a la vida cotidiana de las personas. Casus Revista de Investigación y Casos en Salud. 2017;2(2):80-81.

12. Ministerio de salud. Plan esperanza [Internet]. Lima, Perú: MINSA; 2015 [citado el 28 enero de 2019]. Disponible en: ftp://ftp2.minsa.gob.pe/descargas/ ogc/boletines/Memoria-PlanEsperanzaPERU.pdf

13. Aponte M, Mestanza A, Velásquez J, Farro G. Nivel de conocimiento sobre cáncer de mama y características sociodemográficas en mujeres privadas de su libertad Lima, Perú, 2010. Rev enferm Herediana. 2010;3(2):57-63. 
14. Pilco R. Indicadores trazadores de promoción y prevención de cáncer: factores asociados a la baja cobertura. Centro de salud la esperanza. 2016 [Internet]. TacnaPerú; 2017 [citado el 24 febrero de 2019]. Disponible en: http://ugpc.unjbg.edu.pe/pdfproye cto/29112017232715RinnaPilco20 16 indicadores $\% 20$ trazadores $\% 20 \mathrm{~d}$ e\%20promoci\%C3\%B3n\%20y\%2 Oprevenci\%C3\%B3n\%20de\%20c $\% \mathrm{C} 3 \%$ A1nce.pdf

15. Salvador I, Mercado A, Bravo G Baldeón M, Fornasini M. Factores de riesgo y protección para metaplasia y cáncer gástrico: estudio de casos y controles hospitalarios en Quito-Ecuador. Nutrición Hospitalaria. 2018; 32 (3): 1193-1199.

16. Cordero M, Delgado T, Patiño C. Factores de Riesgo asociados a Cáncer Gástrico Hospital del 20. Uthman O, Jadidi E, Moradi T. Instituto del Cáncer, SOLCA.
Cuenca, 2006-2007. Revista Médica HJCA. 2017;4(1):54-59.

17. Cutipa K . Nivel de conocimientos sobre prevención de cáncer de estómago en usuarios del C.S. Primavera en el distrito El Agustino, 2010 [Tesis de licenciatura]. Lima: Universidad nacional mayor de san marcos; 2011.

18. Ministerio de Salud. A tu salud dile SIS [internet]. Lima, Perú: MINSA; 2011 [citado el 07 febrero de 2019]. Disponible en: http://www.sis.gob.pe/nuevoPortal /iseguros.asp

19. Seguro Social de Salud del Perú Nuestra Institución [Internet]. Lima, Perú: Essalud; 2018 [citado el 09 febrero de 2019]. Disponible en:

http://www.essalud.gob.pe/nuestra -institucion/

Socioeconomic position and incidence of gastric cancer: a systematic review and metaanalysis. Journal Epidemiology Community Health. 2013 67(10):54-60

21. Karimi $P, \quad$ Islami $F$, Anandasabapathy S, Freedman N, Kamangar F. Gastric cancer: descriptive epidemiology, risk factors, screening, and prevention. Cancer Epidemiology and Prevention Biomarkers. 2014; 23(5):700-713.

22. Organización mundial de la salud. Ampliación del rol de las enfermeras y enfermeros en la atención primaria de salud [Internet]. Lima, Perú: OMS; 2018 [citado el 04 marzo de 2020]. Disponible en: https://iris.paho.org/bitstream/hand le/10665.2/34959/9789275320037 _spa.pdf?sequence $=5 \&$ isAllowed $=$ $\mathrm{y}$

23. Ubillús M. Cáncer y la Pobreza están fuertemente correlacionados en el Perú. Revista peruana de ciencias de la salud 2019;1(1):2130. 